\title{
XL-WiC: A Multilingual Benchmark for Evaluating Semantic Contextualization
}

\author{
Alessandro Raganato ${ }^{\star \odot}$ Tommaso Pasini ${ }^{\star \diamond}$ \\ Jose Camacho-Collados* Mohammad Taher Pilehvar ${ }^{\star}$ \\ ${ }^{\curvearrowright}$ Department of Digital Humanities, University of Helsinki, Finland \\ $\diamond$ SapienzaNLP Group, Computer Science Department, Sapienza University of Rome, Italy \\ * School of Computer Science and Informatics, Cardiff University, United Kingdom \\ - Tehran Institute for Advanced Studies, Iran \\ Palessandro.raganato@helsinki.fi, $\gg$ pasini@di.uniromal.it, \\ *camachocollados jecardiff.ac.uk, "mp792@cam.ac.uk
}

\begin{abstract}
The ability to correctly model distinct meanings of a word is crucial for the effectiveness of semantic representation techniques. However, most existing evaluation benchmarks for assessing this criterion are tied to sense inventories (usually WordNet), restricting their usage to a small subset of knowledge-based representation techniques. The Word-in-Context dataset (WiC) addresses the dependence on sense inventories by reformulating the standard disambiguation task as a binary classification problem; but, it is limited to the English language. We put forward a large multilingual benchmark, XL-WiC, featuring gold standards in 12 new languages from varied language families and with different degrees of resource availability, opening room for evaluation scenarios such as zero-shot cross-lingual transfer. We perform a series of experiments to determine the reliability of the datasets and to set performance baselines for several recent contextualized multilingual models. Experimental results show that even when no tagged instances are available for a target language, models trained solely on the English data can attain competitive performance in the task of distinguishing different meanings of a word, even for distant languages. XL-WiC is available at https://pilehvar.github.io/xlwic/.
\end{abstract}

\section{Introduction}

One of the desirable properties of contextualized models, such as BERT (Devlin et al., 2019) and its derivatives, lies in their ability to associate dynamic representations to words, i.e., embeddings that can change depending on the context. This provides the basis for the model to distinguish different meanings (senses) of words without the need to resort to an explicit sense disambiguation step. The conventional evaluation framework for this property has

Authors marked with a star $\left(^{\star}\right)$ contributed equally. been Word Sense Disambiguation (Navigli, 2009, WSD). However, evaluation benchmarks for WSD are usually tied to external sense inventories (often WordNet (Fellbaum, 1998)), making it extremely difficult to evaluate systems that do not explicitly model sense distinctions in the inventory, effectively restricting the benchmark to inventory-based sense representation techniques and WSD systems. This prevents a direct evaluation of lexical semantic capacity for a wide range of inventory-free models, such as the dominating language model-based contextualized representations.

Pilehvar and Camacho-Collados (2019) addressed this dependence on sense inventories by reformulating the WSD task as a simple binary classification problem: given a target word $w$ in two different contexts, $c_{1}$ and $c_{2}$, the task is to identify if the same meaning (sense) of $w$ was intended in both $c_{1}$ and $c_{2}$, or not. The task was framed as a dataset, called Word-in-Context (WiC), which is also a part of the widely-used SuperGLUE benchmark (Wang et al., 2019). Despite allowing a significantly wider range of models for direct WSD evaluations, WiC is limited to the English language only, preventing the evaluation of models in other languages and in cross-lingual settings.

In this paper, we present a new evaluation benchmark, called XL-WiC, that extends the WiC dataset to 12 new languages from different families and with different degrees of resource availability: Bulgarian (BG), Chinese (ZH), Croatian (HR), Danish (DA), Dutch (NL), Estonian (ET), Farsi (FA), French (FR), German (DE), Italian (IT), Japanese (JA) and Korean (KO). With over $80 \mathrm{~K}$ instances, our benchmark can serve as a reliable evaluation framework for contextualized models in a wide range of heterogeneous languages. XL-WiC can also serve as a suitable testbed for cross-lingual experimentation in settings such as zero-shot or 
few-shot transfer across languages. As an additional contribution, we tested several pretrained multilingual models on XL-WiC, showing that they are generally effective in transferring sense distinction knowledge from English to other languages in the zero-shot setting. However, with more training data at hand for target languages, monolingual approaches gain ground, outperforming their multilingual counterparts by a large margin.

\section{Related Work}

$\mathrm{XL}-\mathrm{WiC}$ is a benchmark for inventory-independent evaluation of WSD models (Section 2.1), while the multilingual nature of the dataset makes it an interesting resource for experimenting with crosslingual transfer (Section 2.2).

\subsection{Word Sense Disambiguation}

The ability to identify the intended sense of a polysemous word in a given context is one of the fundamental problems in lexical semantics. It is usually addressed with two different kinds of approaches relying on either sense-annotated corpora (Bevilacqua and Navigli, 2020; Scarlini et al., 2020; Blevins and Zettlemoyer, 2020) or knowledge bases (Moro et al., 2014; Agirre et al., 2014; Scozzafava et al., 2020). Both are usually evaluated on dedicated benchmarks, including at least five WSD tasks in Senseval and SemEval series, from 2001 (Edmonds and Cotton, 2001) to 2015 (Moro and Navigli, 2015a) that are included in the Raganato et al. (2017)'s test suite. All these tasks are framed as classification problems, where disambiguation of a word is defined as selecting one of the predefined senses of the word listed by a sense inventory. This brings about different limitations such as restricting senses only to those defined by the inventory, or forcing the WSD system to explicitly model sense distinctions at the granularity level defined by the inventory.

Stanford Contextual Word Similarity (Huang et al., 2012) is one of the first datasets that focuses on ambiguity but outside the boundaries of sense inventories, and as a similarity measurement between two words in their contexts. Pilehvar and Camacho-Collados (2019) highlighted some of the limitations of the dataset that prevent a reliable evaluation, and proposed the Word-in-Context (WiC) dataset. $\mathrm{WiC}$ is the closest dataset to ours, which provides around 10K instances (1400 instances for 1184 unique target nouns and verbs in the test set), but for the English language only.

\subsection{Cross-lingual NLP}

A prerequisite for research on a language is the availability of relevant evaluation benchmarks. Given its importance, construction of multilingual datasets has always been considered as a key contribution in NLP research and numerous benchmarks exist for a wide range of tasks, such as semantic parsing (Hershcovich et al., 2019), word similarity (Camacho-Collados et al., 2017; Barzegar et al., 2018), sentence similarity (Cer et al., 2017), or WSD (Navigli et al., 2013; Moro and Navigli, 2015b). A more recent example is XTREME (Hu et al., 2020), a benchmark that covers around 40 languages in nine syntactic and semantic tasks.

On the other hand, pre-trained language models have recently proven very effective in transferring knowledge in cross-lingual NLP tasks (Devlin et al., 2019; Conneau et al., 2020). This has further magnified the requirement for rigorous multilingual benchmarks that can be used as basis for this direction of research (Artetxe et al., 2020b).

\section{XL-WiC: The Benchmark}

In this section, we describe the procedure we followed to construct the XL-WiC benchmark. Our framework is based on the original WiC dataset, which we extend to multiple languages.

\subsection{English WiC}

Each instance of the original WiC dataset (Pilehvar and Camacho-Collados, 2019) is composed of a target word (e.g., justify) and two sentences where the target word occurs (e.g., "Justify the margins" and "The end justifies the means"). The task is a binary classification: to decide whether the same sense of the target word (justify) was intended in the two contexts or not. The dataset was built using example sentences from resources such as Wiktionary, WordNet (Miller, 1995) and VerbNet (Schuler et al., 2009).

\subsection{XL-WiC}

We followed Pilehvar and Camacho-Collados (2019) and constructed XL-WiC based on example usages of words in sense inventories. Example usages are curated in a way to be self contained and clearly distinguishable across different senses of a word; hence, they provide a reliable basis for the binary classification task. Specifically, for a word 


\begin{tabular}{|c|c|c|c|c|}
\hline Lang. & Target Word & Sentence 1 & Sentence 2 & Label \\
\hline $\mathrm{EN}$ & Beat & We beat the competition. & Agassi beat Becker in the tennis championship. & True \\
\hline DA & Tro & Jeg tror på det, min mor fortalte. & Maria troede ikke sine egne $\varnothing$ jne. & True \\
\hline ET & Ruum & Ühel hetkel olin väljaspool aega ja ruumi. & Ümberringi oli lõputu tühi ruum. & True \\
\hline FR & Causticité & Sa causticité lui a fait bien des ennemis. & La causticité des acides. & False \\
\hline KO & 틀림 & 틀림이 있는지 없는지 세어 보시오. & 그 아이 하는 짓에 틀림이 있다면 모두 이 어미 죄이지요. & False \\
\hline $\mathrm{ZH}$ & 發 & 建築師希望發大火燒掉城市的三分之一。 & 如果南美洲氣壓偏低,則印度可能發乾旱 & True \\
\hline FA & صرف & صرف غذا نيم ساعت طول كشيد. & معلم صرف افعال ماضى عربى را آموزش داد. & False \\
\hline
\end{tabular}

Table 1: Sample instances from XL-WiC for different languages.

$w$ and for all its senses $\left\{s_{1}^{w}, \ldots, s_{n}^{w}\right\}$, we extract from the inventory all the example usages. We then pair those examples that correspond to the same sense $s_{i}^{w}$ to form a positive instance (True label) while examples from different senses (i.e., $s_{i}^{w}$ and $s_{j}^{w}$ where $i \neq j$ ) are paired as a negative instance (False label).

We leveraged two main sense inventories for this extension: Multilingual WordNet (Section 3.2.1) and Wiktionary (Section 3.2.2).

\subsubsection{Multilingual WordNet}

WordNet (Miller, 1995) is the de facto sense inventory for English WSD. The resource was originally built as an English lexical database in 1995, but since then there have been many efforts to extend it to other languages (Bond and Paik, 2012). We took advantage of these extensions to construct XL-WiC. In particular, we processed the WordNet versions of Bulgarian (Simov and Osenova, 2010), Chinese (Huang et al., 2010), Croatian (Raffaelli et al., 2008), Danish (Pedersen et al., 2009), Dutch (Postma et al., 2016), Estonian (Vider and Orav, 2002), Japanese (Isahara et al., 2008), Korean (Yoon et al., 2009) and Farsi (Shamsfard et al., 2010). ${ }^{1}$

Farsi: Semi-automatic extraction. FarsNet v3.0 (Shamsfard et al., 2010) comprises 30K synsets with over $100 \mathrm{~K}$ word entries. Many of these synsets are mapped to the English database; however, each synset provides just one example usage for a target word. This prevents us from applying the automatic extraction of positive examples. Therefore, we utilized a semi-automatic procedure for the construction of the Farsi set. To this end, for each word, we extracted all example usages from

\footnotetext{
${ }^{1}$ We tried other WordNet versions such as Albanian (Ruci, 2008), Basque (Pociello et al., 2008), Catalan (Benítez et al., 1998), Galician (Guinovart, 2011), Hungarian (Miháltz et al., 2008), Italian (Pianta et al., 2002), Slovenian (Fišer et al., 2012) and Spanish (Atserias et al., 2004); but, they did not contain enough examples.
}

FarsNet, and asked an annotator to group them into positive and negative pairs. The emphasis was to make a challenging dataset with sense distinctions that are easily interpretable by humans. This can also be viewed as a case study to understand the real gap between human and machine performance in settings where manual curation of instances is feasible.

Filtering. WordNet is often considered to be a fine-grained resource, especially for verbs (Duffield et al., 2007). In some cases, the exact meaning of a word can be hard to assess, even for humans. For example, WordNet lists 29 distinct meanings for the noun line, two of which correspond to the horizontally and the vertically organized line formations. Therefore, to cope with this issue, we followed Pilehvar and Camacho-Collados (2019) and filtered out all pairs whose target senses were connected by an edge (including sister-sense relations) in WordNet's semantic network or if they belonged to the same supersense, i.e., one of the 44 lexicographer files ${ }^{2}$ in WordNet which cluster concepts into semantic categories, e.g., Animal, Cognition, Food, etc. For example, the Japanese instance "成長 中の企業は大な指導者いなければならない” ("Growing companies must have bold leaders"), “彼は安定した大きな企業に投資するだけだ” ("He just invested in big stable companies") for the target word “企業” (“company”) is discarded as its corresponding synsets, i.e., "An organization created for business ventures" and "An institution created to conduct business", are grouped under the same supersense in WordNet, i.e., Group.

Finally, all datasets are split into development ${ }^{3}$ and test. At the end of this step, we ensure that both test and development sets have the same number of positive and negative instances. An excerpt of examples included in some of our datasets are

\footnotetext{
${ }^{2}$ wordnet.princeton.edu/documentation/lexnames5wn

${ }^{3}$ Development sets are intended to be used for different purposes, such as training or validation, as we will show in our experiments in Section 5.1.
} 


\begin{tabular}{|c|c|c|c|c|c|c|c|c|c|c|c|c|c|c|}
\hline \multirow{2}{*}{ Split } & \multirow{2}{*}{ Stat } & \multirow{2}{*}{$\frac{\mathrm{WiC}}{\mathrm{EN}}$} & \multicolumn{9}{|c|}{ Multilingual WordNet } & \multicolumn{3}{|c|}{ Wiktionary } \\
\hline & & & BG & DA & ET & FA & HR & JA & KO & NL & $\mathbf{Z H}$ & DE & FR & IT \\
\hline \multirow{3}{*}{ Train } & Instances & 5428 & - & - & - & - & - & - & - & - & - & 48042 & 39428 & 1144 \\
\hline & Unique Words & 1265 & - & - & - & - & - & - & - & - & - & 23213 & 20221 & 721 \\
\hline & Avg. Context Len & 16.8 & - & - & - & - & - & - & - & - & - & 32.7 & 32.3 & 23.2 \\
\hline \multirow{3}{*}{ Dev } & Instances & 638 & 998 & 852 & 98 & 200 & 104 & 208 & 404 & 250 & 3046 & 8870 & 8588 & 198 \\
\hline & Unique Words & 599 & 354 & 542 & 63 & 174 & 82 & 137 & 183 & 150 & 867 & 4383 & 3517 & 136 \\
\hline & Avg. Context Len & 17.1 & 8.4 & 32.6 & 19.8 & 24.8 & 17.9 & 20.8 & 5.7 & 20.1 & 45.7 & 32.5 & 34.3 & 23.2 \\
\hline \multirow{3}{*}{ Test } & Instances & 1400 & 1220 & 3406 & 390 & 800 & 408 & 824 & 1014 & 1004 & 5538 & 24268 & 22232 & 592 \\
\hline & Unique Words & 1184 & 567 & 2088 & 276 & 533 & 305 & 476 & 475 & 600 & 1888 & 11734 & 3517 & 394 \\
\hline & Avg. Context Len & 17.2 & 8.5 & 32.6 & 19.4 & 23.5 & 18.1 & 20.6 & 6.0 & 19.9 & 46.0 & 32.9 & 36.4 & 23.4 \\
\hline
\end{tabular}

Table 2: Statistics for WordNet and Wiktionary datasets for different languages.

shown in Table 1.

\subsubsection{Wiktionary}

Wiktionary is one of the richest free collaborative lexical databases, available for dozens of languages. In this online resource, each word is provided with definitions for its various potential meanings, some of which are paired with example usages. However, each language has a specific format, and therefore the compilation of these examples requires a careful language-specific parsing. We extracted examples for three European languages for which we did not have WordNet-based data, namely French, German, and Italian. ${ }^{4}$ Once these examples were compiled, the process to build the final dataset was analogous to that for the WordNet-based datasets (see Section 3.2.1), except for the filtering step, which was not feasible as Wiktionary entries are not connected through paradigmatic relations as in WordNet.

For the case of Wiktionary, the number of examples was considerably higher; therefore, we also compiled language-specific training sets, which enabled a comparison between cross-lingual and monolingual models (see Section 5.2). All Wiktionary datasets are split into balanced training, development and test splits, in each of which there are equal number of positive and negative instances.

\subsection{Statistics}

Table 2 shows the statistics of all datasets, including the total number of instances, unique words, and the context length average. ${ }^{5}$ Wiktionary-based

\footnotetext{
${ }^{4}$ French WordNet, WoNeF (Pradet et al., 2014), is built automatically; German WordNet, GermaNet (Hamp and Feldweg, 1997), has a very restrictive license; and the Italian WordNet (Pianta et al., 2002) provides too few examples.

${ }^{5}$ We used the multilingual Stanford NLP toolkit, Stanza (Qi et al., 2020), with the available pre-trained neural models.
}

datasets are substantially larger than the WordNetbased ones, and also provide training sets. The Chinese datasets feature longer contexts on average and contain the largest number of development and testing instances among WordNet-based datasets. Korean, on the other hand, is the one with the shortest contexts, which is expected given its agglutinative nature. As for the training corpora, German and French datasets contain almost ten times the number of instances in the English training set. This allows us to perform a large-scale comparison between cross-lingual and monolingual settings (see Section 5.2) as well as a few-shot analysis (Section 6.2).

\subsection{Validation and human performance}

To verify the reliability of the datasets, we carried out manual evaluation for those languages for which we had access to annotators. To this end, we presented a set of 100 randomly sampled instances from each dataset to the corresponding annotator in the target language. ${ }^{6}$ Annotators were all native speakers of the target language with high-level education. They were provided with a minimal guideline: a brief explanation of their task and a few tagged examples. We did not provide any lexical resource (or any other detailed instructions) to the annotators with the emphasis to make a challenging dataset with sense distinctions that are easily interpretable to the layman. Given an instance, i.e., a pair of sentences containing the same target word, their task consisted of tagging it with a True or False label, depending on the the intended meanings of the word in the two contexts.

Table 3 reports human performance for eight

\footnotetext{
${ }^{6}$ For Farsi, three checker annotators were involved in the validation, each annotating the 100 -instance subset. In this case, we report the average accuracy.
} 


\begin{tabular}{|c|c|c|c|c|c|c|c|c|}
\hline \multirow{2}{*}{$\frac{\text { WiC }}{\text { EN }}$} & \multicolumn{6}{|c|}{ WordNet } & \multicolumn{2}{|c|}{ Wiktionary } \\
\hline & DA & FA & IT & $\mathbf{J A}$ & KO & $\mathbf{Z H}$ & DE & IT \\
\hline $80.0^{*}$ & 87.0 & 97.0 & 82.0 & 75.0 & 76.0 & 85.0 & 74.0 & 78.0 \\
\hline
\end{tabular}

Table 3: Human performance (in terms of accuracy) for different languages in $\mathrm{XL}-\mathrm{WiC}$. *From the original English WiC dataset.

datasets in XL-WiC. All accuracy figures are around $80 \%$, i.e., in the same ballpark as the original WiC English dataset, which attests the reliability of underlying resources and the construction procedure. The only exception is for Farsi, for which the checker annotators agree with the gold labels in $97 \%$ of the instances (by average). This corroborates our emphasis on the annotation procedure for this manually-created dataset to have sense distinctions that are easily interpretable by humans. As for Wiktionary, the human agreements are lower than those for the WordNet counterparts. ${ }^{7}$ This was partly expected given that the semantic network-based filtering step (see Section 3.2.1) was not feasible for the case of Wiktionary datasets due to the nature of the underlying resource.

\section{Experimental Setup}

For our experiments, we implemented a simple, yet effective, baseline based on a Transformer-based text encoder (Vaswani et al., 2017) and a logistic regression classifier, following Wang et al. (2019). The model takes as input the two contexts and first tokenizes them, splitting the input words into subtokens. The encoded representations of the target words are concatenated and fed to the logistic classifier. For those cases where the target word was split by the tokenizer into multiple sub-tokens, we followed Devlin et al. (2019) and considered the representation of its first sub-token.

As regards the text encoder, we carried out the experiments with three different multilingual models, i.e., the multilingual version of BERT (Devlin et al., 2019) (mBERT) and the base and large versions of XLM-RoBERTa (Conneau et al., 2020) (XLMR-base and XLMR-large, respectively). In the monolingual setting, we used the following language-specific models: BERT-de ${ }^{8}$,

\footnotetext{
${ }^{7}$ Even if not part of XL-WiC, we also compiled and validated a small Italian WordNet dataset to compare it with its Wiktionary counterpart. Table 8 in Appendix includes an additional table comparing the nature of these two datasets (including zero-shot cross-lingual transfer results).

${ }^{8}$ huggingface.co/dbmdz/bert-base-german-cased
}

CamemBERT-large (Martin et al., 2020) ${ }^{9}$, BERTit $^{10}$, and ParsBERT ${ }^{11}$ (Farahani et al., 2020), respectively, for German, French, Italian, and Farsi. As for all the other languages covered by the WordNet datasets, i.e., Bulgarian, Chinese, Croatian, Danish, Dutch, Estonian, Japanese and Korean, we used the pre-trained models made available by TurkuNLP. ${ }^{12}$ We refer to each language-specific model as L-BERT.

In all experiments we trained the baselines to minimize the binary cross-entropy loss between their prediction and the gold label with the Adam (Kingma and $\mathrm{Ba}$, 2015) optimizer. Training is carried out for 10 epochs with the learning rate fixed to $1 e^{-5}$ and weight decay set to 0 . As for tuning, results are reported for the best training checkpoint (among the 10 epochs) according to the performance on the development set.

\subsection{Evaluation settings}

We evaluated the baselines with different configuration setups, depending on the data used for training and tuning.

Cross-Lingual Zero-shot. This setting aims at assessing the capabilities of multilingual models in transferring knowledge captured in the English language to other languages. As training set, we used the English training set of WiC. As for tuning, depending on the setting, we either used the English development set of $\mathrm{WiC}$ or language-specific development sets of XL-WiC (Section 3.2.1). We report results on all WordNet and Wiktionary datasets of XL-WiC, i.e., Bulgarian, Chinese, Croatian, Danish, Dutch, Estonian, Farsi, French, German, Italian, Japanese and Korean.

Multilingual Fine-Tuning. In this setting, models are first trained on the WiC's English training set, and then further fine-tuned on the development sets of the target languages in XL-WiC. Depending on the training set used, we report results for two configurations: (i) EN+Target Language, combining with WiC's training data and the language-specific WordNet development sets for each language, and (ii) EN+All Languages, combining that with all WordNet development sets for all languages in XL-WiC, merged as one dataset.

\footnotetext{
${ }^{9}$ huggingface.co/camembert/camembert-large

${ }^{10}$ huggingface.co/dbmdz/bert-base-italian-xxl-cased

${ }^{11}$ github.com/hooshvare/parsbert

${ }^{12}$ github.com/TurkuNLP/wikibert
} 


\begin{tabular}{lccccccccc}
\hline Model & BG & DA & ET & FA & HR & JA & KO & NL & ZH \\
\hline & & & & Zero-shot cross-lingual setting & Train: EN & - Dev: EN \\
\hline mBERT & 58.28 & 64.86 & 62.56 & 71.50 & 63.97 & 62.26 & 59.76 & 63.84 & 69.36 \\
XLMR-base & 60.73 & 64.79 & 62.82 & 69.88 & 62.01 & 60.44 & 66.96 & 65.73 & 65.78 \\
XLMR-large & $\mathbf{6 6 . 4 8}$ & $\mathbf{7 1 . 1 1}$ & $\mathbf{6 8 . 7 1}$ & $\mathbf{7 5 . 2 5}$ & $\mathbf{7 2 . 3 0}$ & 63.83 & $\mathbf{6 9 . 6 3}$ & $\mathbf{7 2 . 8 1}$ & $\mathbf{7 3 . 1 5}$ \\
\hline \hline & & \multicolumn{7}{c}{ Test instances translated to English } & Train: EN - Dev: EN \\
\hline mBERT & 63.52 & 62.71 & 68.46 & - & 60.54 & 63.95 & - & 66.53 & - \\
XLMR-base & 60.98 & 60.24 & 62.82 & - & 60.78 & 61.77 & - & 64.64 & - \\
XLMR-large & 64.43 & 66.64 & 63.84 & - & 69.85 & 64.44 & - & 72.11 & - \\
L-BERT & 64.02 & 65.38 & 64.62 & - & 69.61 & $\mathbf{6 5 . 9 0}$ & - & 68.43 & - \\
\hline \multicolumn{1}{c}{ Train and Dev instances translated from English to target language } & Train: T-EN & - Dev: T-EN \\
\hline mBERT & 56.97 & 60.25 & 59.48 & - & 66.91 & 58.13 & - & 60.06 & - \\
XLMR-base & 56.07 & 52.85 & 57.18 & - & 64.22 & 56.19 & - & 60.56 & - \\
XLMR-large & 62.13 & 63.39 & 64.87 & - & 66.18 & 59.47 & - & 66.73 & - \\
L-BERT & 54.26 & 60.57 & 59.49 & - & 61.52 & 58.98 & - & 60.46 & - \\
\hline
\end{tabular}

Table 4: Results on the WordNet test sets when using only English training data in WiC, either in zero-shot crosslingual setting (top block) or translation-based settings (the lower two blocks). T-EN is a target language dataset, automatically constructed by translating English instances in WiC.

Monolingual. In this setting, we trained each model on the corresponding training set of the target language only. For the case of WordNet datasets (where no training sets are available), we used the development sets for training. In this case we split each development set into two subsets with 9:1 ratio (for training and development). As for the Wiktionary datasets, we used the corresponding training and development sets for each language (Section 3.2.2).

Translation. In this last setting we make use of existing neural machine translation (NMT) models to translate either the training or the test set, essentially reducing the cross-lingual problem to a monolingual one. In particular, we used the general-domain translation models from the Opus-MT project ${ }^{13}$ (Tiedemann and Thottingal, 2020) available for the following language pairs: English-Bulgarian, English-Croatian, EnglishDanish, English-Dutch, English-Estonian, and English-Japanese. The models are trained on all OPUS parallel corpora collection (Tiedemann, 2012), using the state-of-the-art 6-layer Transformer-based architecture (Vaswani et al., 2017). ${ }^{14}$ In this configuration, as the original target word may be lost during automatic translation, we view the task as context (sentence) similarity

\footnotetext{
${ }^{13}$ github.com/Helsinki-NLP/Opus-MT

${ }^{14}$ More details about the NMT models and their translation quality are given in the Appendix (Table 13).
}

as proposed by Pilehvar and Camacho-Collados (2019). ${ }^{15}$ Therefore, for each model, the context vector is given by the start sentence symbol. We note that while training custom optimized NMT models for each target language may result in better overall performance, this is beyond the scope of this work.

Evaluation Metrics. Since all datasets are balanced, we only report accuracy, i.e., the ratio of correctly predicted instances (true positives or true negatives) to the total number of instances.

\section{Results}

In this section, we report the results for the configurations discussed in the previous section on the XL-WiC benchmark. We organize the experiments into two parts, based on the test dataset: WordNet (Section 5.1) and Wiktionary (Section 5.2).

\subsection{WordNet datasets}

Using English data only. Table 4 shows results on the XL-WiC WordNet test sets, when only WiC's English data was used for training and tuning purposes. Across the board, XLMR-large consistently achieves the best results, while mBERT and XLMR-base attain scores in the same ballpark. Indeed, the massive pretraining and the number of

\footnotetext{
${ }^{15}$ The Appendix (Table 12) includes another translation baseline that uses dictionary alignments to identify the target word as comparison, but performed worse overall.
} 


\begin{tabular}{lccccccccccc}
\hline Model & EN & BG & DA & ET & FA & HR & JA & KO & NL & ZH \\
\hline & & & & & \multicolumn{5}{c}{ Train: $E N$} & - Dev: Target Language \\
\hline mBERT & - & 59.18 & 64.59 & 63.08 & 70.38 & 64.95 & 59.95 & 63.31 & 64.04 & 70.48 \\
XLMR-base & - & 60.74 & 64.80 & 60.77 & 67.75 & 62.50 & 57.65 & 66.96 & 61.85 & 65.78 \\
XLMR-large & - & $\mathbf{6 6 . 4 8}$ & $\mathbf{7 1 . 1 0}$ & $\mathbf{6 8 . 7 2}$ & $\mathbf{7 3 . 6 3}$ & $\mathbf{7 2 . 3 0}$ & $\mathbf{6 0 . 9 2}$ & $\mathbf{6 9 . 6 3}$ & $\mathbf{6 9 . 6 2}$ & $\mathbf{7 3 . 1 5}$ \\
\hline & & & & & \multicolumn{5}{c}{ Train: EN+Target Language - Dev: EN } \\
\hline mBERT & - & 71.72 & 62.62 & 63.08 & 69.38 & $\mathbf{7 2 . 3 0}$ & 60.92 & 70.91 & 62.95 & 76.72 \\
XLMR-base & - & 64.51 & 64.45 & 60.00 & 65.38 & 71.57 & 58.37 & 65.68 & 64.54 & 73.46 \\
XLMR-large & - & $\mathbf{7 5 . 4 1}$ & $\mathbf{7 0 . 5 2}$ & $\mathbf{6 8 . 9 7}$ & $\mathbf{7 3 . 7 5}$ & 69.61 & $\mathbf{6 3 . 1 1}$ & $\mathbf{7 3 . 4 7}$ & $\mathbf{7 4 . 5 0}$ & $\mathbf{7 7 . 5 2}$ \\
\hline & & & & & \multicolumn{6}{c}{ Train: EN+All Languages - Dev: EN } \\
\hline mBERT & - & 73.03 & 65.09 & 62.31 & 73.63 & 72.30 & 65.53 & 71.01 & 67.73 & 76.53 \\
XLMR-base & - & 67.30 & 67.62 & 59.49 & 64.50 & 66.18 & 57.77 & 67.06 & 66.33 & 71.02 \\
XLMR-large & - & $\mathbf{7 8 . 4 4}$ & $\mathbf{7 1 . 4 9}$ & $\mathbf{7 2 . 0 5}$ & $\mathbf{7 8 . 2 5}$ & $\mathbf{7 6 . 9 6}$ & $\mathbf{6 6 . 3 8}$ & $\mathbf{7 6 . 5 3}$ & $\mathbf{7 7 . 4 9}$ & $\mathbf{7 8 . 9 5}$ \\
\hline & & & & & Train: & Target Language & - Dev: Target Language \\
\hline mBERT & 66.71 & $\mathbf{8 2 . 3 0}$ & 62.13 & 58.21 & 63.75 & 77.45 & $\mathbf{6 1 . 0 4}$ & 70.71 & 64.84 & 76.09 \\
XLMR-base & 64.36 & 79.75 & 64.00 & $\mathbf{6 4 . 3 6}$ & 66.25 & $\mathbf{7 9 . 1 7}$ & 58.86 & 70.61 & 66.33 & 78.11 \\
XLMR-large & 70.14 & 82.05 & $\mathbf{6 6 . 5 3}$ & 59.23 & 68.00 & 76.72 & 55.22 & $\mathbf{7 3 . 0 8}$ & $\mathbf{6 9 . 4 2}$ & $\mathbf{8 1 . 8 3}$ \\
L-BERT & 69.60 & 81.23 & 62.60 & 58.46 & $\mathbf{7 6 . 6 3}$ & 76.47 & 56.07 & 58.68 & 68.73 & 77.36 \\
\hline
\end{tabular}

Table 5: Results on the WordNet test sets when using language-specific data, either for training or for tuning.

parameters of XLMR-large play key roles behind this lead in performance. As regards the translationbased settings (lower two blocks), the performance generally falls slightly behind the zero-shot crosslingual counterpart. This shows that the usage of good quality English data and multilingual models provide a stronger training signal than noisy automatically-translated data. This somehow contrasts with the observations made on other crosslingual tasks in XTREME (Hu et al., 2020), especially in question answering datasets (Artetxe et al., 2020a; Lewis et al., 2020; Clark et al., 2020), where translating data was generally better. This difference could perhaps be reduced with larger monolingual models or accurate alignment, but this would further increase the complexity, and extracting these alignments from NMT models is not trivial (Koehn and Knowles, 2017; Ghader and Monz, 2017; Li et al., 2019).

Utilizing language-specific data. Table 5 shows results for settings where target language-specific data was used for training or tuning. Comparing the results in the top block (where target language data was used for tuning) with the middle two blocks (where target language data was instead used for training) reveals that it is more effective to leverage the target language data for training, rather than using it for tuning only. Overall, it is clear that adding multilingual data during training drastically improves the results in all languages. In this case, training (fine-tuning) is performed on a larger dataset which, despite having examples from different languages, provides a stronger signal to the models, enabling them to better generalize across languages. On the contrary, when only using target language data for training and tuning (last block in the table), results drop for most languages. This highlights the fact that having additional training data is beneficial, reinforcing the utility of multilingual models and cross-lingual transfer.

\subsection{Wiktionary Datasets}

In Table 6 we show results for the Wiktionary datasets. Differently from the results reported for the WordNet datasets (Table 4), models are less effective in the zero-shot setting, performing from 10 to almost 20 points lower than their counterparts trained on data in the target language. This can be attributed to the size of the available training data. Indeed, while in the WordNet datasets we only have a very small amount of data at our disposal for training (see statistics in Table 2), Wiktionary training sets are much larger, hence providing enough data to the models to better generalize. Once again, XLMR-large proves to be the best model in the 


\begin{tabular}{|c|c|c|c|c|}
\hline & Model & DE & FR & IT \\
\hline \multirow{3}{*}{ 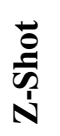 } & mBERT & 58.27 & 56.00 & 58.61 \\
\hline & XLMR-base & 58.30 & 56.13 & 55.91 \\
\hline & XLMR-large & 65.83 & 62.50 & 64.86 \\
\hline \multirow{4}{*}{$\stackrel{\varrho}{e^{\circ}}$} & mBERT & 81.58 & 73.67 & 71.96 \\
\hline & XLMR-base & 80.84 & 73.06 & 68.58 \\
\hline & XLMR-large & 84.03 & 76.16 & 72.30 \\
\hline & L-BERT & 82.90 & 78.14 & 72.64 \\
\hline
\end{tabular}

Table 6: Results on the Wiktionary test sets in different training settings: zero-shot (Z-Shot) and monolingual training (Mono). L-BERT stands for language-specific models, i.e., BERT-de, CamemBERT-large and BERTit for German, French and Italian, respectively.

zero-shot setting and a competitive alternative to the language-specific models (L-BERT row) in the monolingual setting, performing 1.1 points higher in German and 2 and 0.3 lower in French and Italian, respectively.

\section{Analysis}

In this section, we delve into the performance of the models on XL-WiC and analyze relevant aspects about their behaviour.

\subsection{Seen and Unseen Words}

For this analysis, we aim at measuring the difference in performance when a given target word was seen (as a target word) at training time or not. To this end, we evaluate our baselines when trained on the German, French, and Italian Wiktionary training sets and tested on two different subsets of the larger language-specific Wiktionary test sets: In-Vocabulary (IV), containing only the examples whose target word was seen at training time; and Out-of-Vocabulary (OOV), containing only the examples whose target word was not seen during training. We report the results in Table 7.

In general, multilingual models are less reliable when classifying unseen instances, lagging between 1 and 12 points behind in performance, depending on the language and on the model considered. This can be attributed to the fact that their vocabulary is shared among several languages, and therefore may have less knowledge stored about particular words that do not occur often. The performance drop of language-specific models (L-BERT) is less pronounced, with the French architecture (CamemBERT-large) attaining even higher performance ( 0.4 points more) on the OOV set.

\begin{tabular}{llccc}
\hline & Model & DE & FR & IT \\
\hline \multirow{4}{*}{ mBERT } & 81.86 & 72.92 & 73.15 \\
XLMR-base & 81.17 & 71.92 & 70.69 \\
XLMR-large & $\mathbf{8 4 . 2 4}$ & 75.61 & $\mathbf{7 5 . 1 2}$ \\
& L-BERT & 83.23 & $\mathbf{7 7 . 6 2}$ & 73.89 \\
\hline \multirow{3}{*}{ mBERT } & 70.08 & 71.24 & 68.54 \\
O XLMR-base & 71.31 & 71.14 & 62.36 \\
O XLMR-large & 72.54 & 73.93 & 65.17 \\
& L-BERT & $\mathbf{7 6 . 6 4}$ & $\mathbf{7 8 . 0 0}$ & $\mathbf{6 9 . 1 0}$ \\
\hline
\end{tabular}

Table 7: Results on the in-vocabulary (IV) and outof-vocabulary (OOV) Wiktionary test sets. L-BERT stands for each language-specific model, i.e., BERTbase-de, camemBERT and BERT-base-xxl-it for German, French and Italian, respectively.

\subsection{Few-shot Monolingual}

As an additional experiment, we investigate the impact of training size on performance. To this end, we leveraged the Wiktionary datasets for German, French and Italian, which allow us to use varyingsized training sets, and created 7 training sets with $10,25,50,100,250,500,1000$ instances. $^{16}$

The results of this experiment are displayed in Figure 1. When providing only 10 examples, most of the models perform similarly or even worse than random, i.e., $50 \%$ accuracy. In this setting, language-specific models (L-BERT) attain better results than their multilingual counterparts, showing better generalization capabilities when fewer examples are provided. This also goes in line with what we found in the previous experiment on seen and unseen words. With less than $5 \%$ of the training data (1000 instances in French and German and 50 instances in Italian), all models attain roughly $85 \%$ of their performance with full training data, comparable to results reported for the zero-shot setting (Table 6).

\section{Conclusions}

In this paper we have introduced $\mathrm{XL}-\mathrm{WiC}$, a large benchmark for evaluating context-sensitive models. XL-WiC comprises datasets for a heterogeneous set of 13 languages, including the original English data in WiC (Pilehvar and Camacho-Collados, 2019), providing an evaluation framework not only for contextualized models in those languages, but also for experimentation in a cross-lingual transfer setting. Our evaluations show that, even though cur-

\footnotetext{
${ }^{16}$ Bigger datasets are supersets of smaller ones.
} 

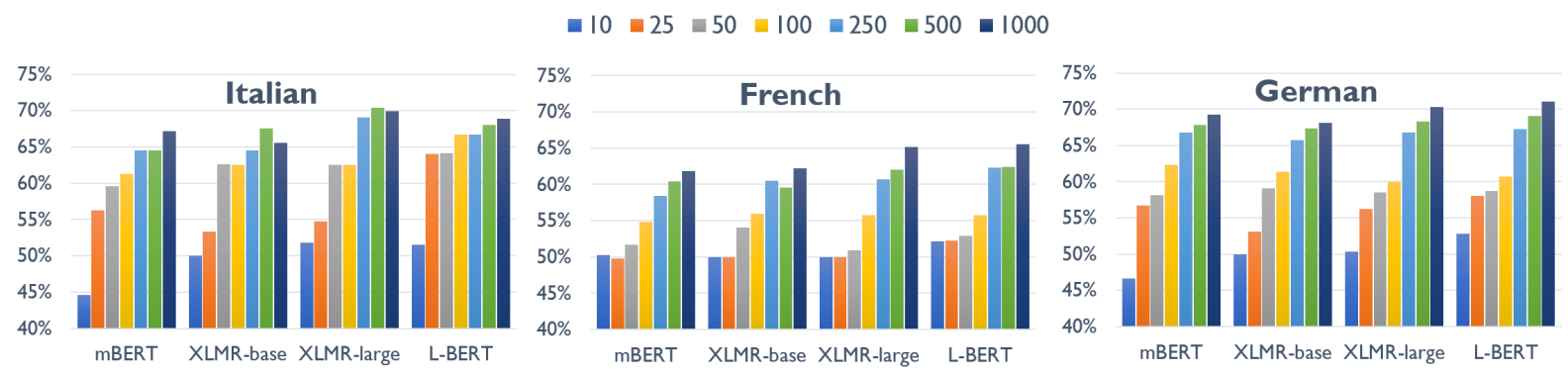

Figure 1: The impact of training set size (\# of instances) on performance, for the Wiktionary datasets.

rent language models are effective performers in the zero-shot cross-lingual setting (where no instances in the target language are provided), there is still room for improvement, especially for far languages such as Japanese or Korean.

As for future work, we plan to investigate using languages other than English for training (e.g., our larger French and German training sets) in our cross-lingual transfer experiments, since English may not always be the optimal source language (Anastasopoulos and Neubig, 2020). Finally, while in our comparative analysis we have focused on a quantitative evaluation for all languages, an additional error analysis per language would be beneficial in revealing the weaknesses and limitations of cross-lingual models.

\section{Acknowledgments}

We would like to thank Qincheng Zhang (Chinese), Janine Siewert (Danish), Houman Mehrafarin, Hossein Mohebbi, and Ali Modarresi (Farsi), Ángela Collados Aís (German), Asahi Ushio (Japanese), and Yunseo Joung (Korean) for their help with the manual evaluation of the datasets.

Alessandro gratefully acknowledges the support of the FoTran project, funded by the European Research Council (ERC) under the European Union's Horizon 2020 research and innovation programme (grant agreement No 771113), and the CSC

erc - IT Center for Science, Finland, for computational resources.

Tommaso gratefully acknowledges the support of the ERC Consolidator Grant MOUSSE No. 726487 under the European Union's Horizon 2020 research and innovation programme.

\section{References}

Eneko Agirre, Oier Lopez de Lacalle, and Aitor Soroa. 2014. Random walks for knowledge-based word sense disambiguation. Computational Linguistics, 40(1):57-84.

Antonios Anastasopoulos and Graham Neubig. 2020. Should all cross-lingual embeddings speak English? In Proceedings of the 58th Annual Meeting of the Association for Computational Linguistics, pages 8658-8679, Online. Association for Computational Linguistics.

Mikel Artetxe, Sebastian Ruder, and Dani Yogatama. 2020a. On the cross-lingual transferability of monolingual representations. In Proceedings of the 58th Annual Meeting of the Association for Computational Linguistics, pages 4623-4637, Online. Association for Computational Linguistics.

Mikel Artetxe, Sebastian Ruder, Dani Yogatama, Gorka Labaka, and Eneko Agirre. 2020b. A call for more rigor in unsupervised cross-lingual learning. In Proceedings of the 58th Annual Meeting of the Association for Computational Linguistics, pages 7375-7388, Online. Association for Computational Linguistics.

Jordi Atserias, Luís Villarejo, and German Rigau. 2004. Spanish WordNet 1.6: Porting the Spanish Wordnet across Princeton versions. In Proceedings of the Fourth International Conference on Language Resources and Evaluation (LREC'04), Lisbon, Portugal. European Language Resources Association (ELRA).

Siamak Barzegar, Brian Davis, Manel Zarrouk, Siegfried Handschuh, and Andre Freitas. 2018. SemR-11: A multi-lingual gold-standard for semantic similarity and relatedness for eleven languages. In Proceedings of the Eleventh International Conference on Language Resources and Evaluation (LREC-2018), Miyazaki, Japan. European Languages Resources Association (ELRA).

Laura Benítez, Sergi Cervell, Gerard Escudero, Mònica López, German Rigau, and Mariona Taulé. 1998. Methods and tools for building the catalan wordnet. Proceedings of ELRA Workshop on Language Resources for European Minority Languages. 
Michele Bevilacqua and Roberto Navigli. 2020. Breaking through the $80 \%$ Glass Ceiling: Raising the State of the Art in Word Sense Disambiguation by Incorporating Knowledge Graph Information. In Proc. of $A C L$, pages 2854-2864.

Terra Blevins and Luke Zettlemoyer. 2020. Moving Down the Long Tail of Word Sense Disambiguation with Gloss Informed Bi-encoders. In Proc. of $A C L$, pages 1006-1017.

Francis Bond and Kyonghee Paik. 2012. A survey of wordnets and their licenses. In GWC 2012 6th International Global Wordnet Conference, volume 8, pages 64-71.

Jose Camacho-Collados, Mohammad Taher Pilehvar, Nigel Collier, and Roberto Navigli. 2017. SemEval2017 task 2: Multilingual and cross-lingual semantic word similarity. In Proceedings of the 11th International Workshop on Semantic Evaluation (SemEval2017), pages 15-26, Vancouver, Canada. Association for Computational Linguistics.

Daniel Cer, Mona Diab, Eneko Agirre, Iñigo LopezGazpio, and Lucia Specia. 2017. SemEval-2017 task 1: Semantic textual similarity multilingual and crosslingual focused evaluation. In Proceedings of the 11th International Workshop on Semantic Evaluation (SemEval-2017), pages 1-14, Vancouver, Canada. Association for Computational Linguistics.

Christos Christodouloupoulos and Mark Steedman. 2015. A massively parallel corpus: the bible in 100 languages. Language resources and evaluation, 49(2):375-395.

Jonathan H Clark, Eunsol Choi, Michael Collins, Dan Garrette, Tom Kwiatkowski, Vitaly Nikolaev, and Jennimaria Palomaki. 2020. Tydi qa: A benchmark for information-seeking question answering in typologically diverse languages. Transactionsof the Association of Computational Linguistics.

Alexis Conneau, Kartikay Khandelwal, Naman Goyal, Vishrav Chaudhary, Guillaume Wenzek, Francisco Guzmán, Edouard Grave, Myle Ott, Luke Zettlemoyer, and Veselin Stoyanov. 2020. Unsupervised cross-lingual representation learning at scale. In Proceedings of the 58th Annual Meeting of the Association for Computational Linguistics, pages 8440 8451, Online. Association for Computational Linguistics.

Jacob Devlin, Ming-Wei Chang, Kenton Lee, and Kristina Toutanova. 2019. Bert: Pre-training of deep bidirectional transformers for language understanding. In Proceedings of the 2019 Conference of the North American Chapter of the Association for Computational Linguistics: Human Language Technologies, Volume 1 (Long and Short Papers), pages 4171-4186.

Cecily Jill Duffield, Jena D Hwang, Susan Windisch Brown, Dmitriy Dligach, Sarah Vieweg, Jenny
Davis, and Martha Palmer. 2007. Criteria for the manual grouping of verb senses. In Proceedings of the Linguistic Annotation Workshop, pages 49-52.

Philip Edmonds and Scott Cotton. 2001. SENSEVAL2: Overview. In Proceedings of SENSEVAL2 Second International Workshop on Evaluating Word Sense Disambiguation Systems, pages 1-5, Toulouse, France. Association for Computational Linguistics.

Mehrdad Farahani, Mohammad Gharachorloo, Marzieh Farahani, and Mohammad Manthouri. 2020. Parsbert: Transformer-based model for persian language understanding.

Christiane Fellbaum, editor. 1998. WordNet: An Electronic Database. MIT Press, Cambridge, MA.

Darja Fišer, Jernej Novak, and Tomaž Erjavec. 2012. slownet 3.0: development, extension and cleaning. In Proceedings of 6th International Global Wordnet Conference (GWC 2012), pages 113-117.

Hamidreza Ghader and Christof Monz. 2017. What does attention in neural machine translation pay attention to? In Proceedings of the Eighth International Joint Conference on Natural Language Processing (Volume 1: Long Papers), pages 30-39, Taipei, Taiwan. Asian Federation of Natural Language Processing.

Xavier Gómez Guinovart. 2011. Galnet: Wordnet 3.0 do galego. Linguamática, 3(1):61-67.

Birgit Hamp and Helmut Feldweg. 1997. Germanet-a lexical-semantic net for german. In Automatic information extraction and building of lexical semantic resources for NLP applications.

Daniel Hershcovich, Zohar Aizenbud, Leshem Choshen, Elior Sulem, Ari Rappoport, and Omri Abend. 2019. SemEval-2019 task 1: Cross-lingual semantic parsing with UCCA. In Proceedings of the 13th International Workshop on Semantic Evaluation, pages 1-10, Minneapolis, Minnesota, USA. Association for Computational Linguistics.

Junjie Hu, Sebastian Ruder, Aditya Siddhant, Graham Neubig, Orhan Firat, and Melvin Johnson. 2020. XTREME: A massively multilingual multitask benchmark for evaluating cross-lingual generalization. Proceedings of the 37th International Conference on Machine Learning (ICML).

Chu-Ren Huang, Shu-Kai Hsieh, Jia-Fei Hong, YunZhu Chen, I-Li Su, Yong-Xiang Chen, and ShengWei Huang. 2010. Chinese Wordnet: Design, Implementation and Application of an Infrastructure for Cross-Lingual Knowledge Processing. Journal of Chinese Information Processing, 24(2):14.

Eric Huang, Richard Socher, Christopher Manning, and Andrew Ng. 2012. Improving word representations via global context and multiple word prototypes. In Proceedings of the 50th Annual Meeting of 
the Association for Computational Linguistics (Volume 1: Long Papers), pages 873-882, Jeju Island, Korea. Association for Computational Linguistics.

Hitoshi Isahara, Francis Bond, Kiyotaka Uchimoto, Masao Utiyama, and Kyoko Kanzaki. 2008. Development of the Japanese WordNet. In Sixth International conference on Language Resources and Evaluation.

Marcin Junczys-Dowmunt, Roman Grundkiewicz, Tomasz Dwojak, Hieu Hoang, Kenneth Heafield, Tom Neckermann, Frank Seide, Ulrich Germann, Alham Fikri Aji, Nikolay Bogoychev, André F. T. Martins, and Alexandra Birch. 2018. Marian: Fast neural machine translation in $\mathrm{C}++$. In Proceedings of ACL 2018, System Demonstrations, pages 116 121, Melbourne, Australia. Association for Computational Linguistics.

Diederik P. Kingma and Jimmy Ba. 2015. Adam: A method for stochastic optimization. In $3 r d$ International Conference on Learning Representations, ICLR 2015, San Diego, CA, USA, May 7-9, 2015, Conference Track Proceedings.

Philipp Koehn and Rebecca Knowles. 2017. Six challenges for neural machine translation. In Proceedings of the First Workshop on Neural Machine Translation, pages 28-39, Vancouver. Association for Computational Linguistics.

Patrick Lewis, Barlas Oguz, Ruty Rinott, Sebastian Riedel, and Holger Schwenk. 2020. MLQA: Evaluating cross-lingual extractive question answering. In Proceedings of the 58th Annual Meeting of the Association for Computational Linguistics, pages 73157330, Online. Association for Computational Linguistics.

Xintong Li, Guanlin Li, Lemao Liu, Max Meng, and Shuming Shi. 2019. On the word alignment from neural machine translation. In Proceedings of the 57th Annual Meeting of the Association for Computational Linguistics, pages 1293-1303, Florence, Italy. Association for Computational Linguistics.

Louis Martin, Benjamin Muller, Pedro Javier Ortiz Suárez, Yoann Dupont, Laurent Romary, Éric de la Clergerie, Djamé Seddah, and Benoît Sagot. 2020. CamemBERT: a tasty French language model. In Proceedings of the 58th Annual Meeting of the Association for Computational Linguistics, pages 7203-7219, Online. Association for Computational Linguistics.

Márton Miháltz, Csaba Hatvani, Judit Kuti, György Szarvas, János Csirik, Gábor Prószéky, and Tamás Váradi. 2008. Methods and Results of the Hungarian WordNet Project. In Proceedings of The Fourth Global WordNet Conference, pages 311-321.

George A Miller. 1995. WordNet: a lexical database for english. Communications of the ACM, 38(11):39-41.
Andrea Moro and Roberto Navigli. 2015a. SemEval2015 task 13: Multilingual all-words sense disambiguation and entity linking. In Proceedings of the 9th International Workshop on Semantic Evaluation (SemEval 2015), pages 288-297, Denver, Colorado. Association for Computational Linguistics.

Andrea Moro and Roberto Navigli. 2015b. Semeval2015 task 13: Multilingual all-words sense disambiguation and entity linking. Proceedings of SemEval-2015.

Andrea Moro, Alessandro Raganato, and Roberto Navigli. 2014. Entity Linking meets Word Sense Disambiguation: a Unified Approach. Transactions of the Association for Computational Linguistics (TACL), 2:231-244.

Roberto Navigli. 2009. Word Sense Disambiguation: A survey. ACM Computing Surveys, 41(2):1-69.

Roberto Navigli, David Jurgens, and Daniele Vannella. 2013. SemEval-2013 task 12: Multilingual word sense disambiguation. In Second Joint Conference on Lexical and Computational Semantics (*SEM), Volume 2: Proceedings of the Seventh International Workshop on Semantic Evaluation (SemEval 2013), pages 222-231, Atlanta, Georgia, USA. Association for Computational Linguistics.

Roberto Navigli and Simone Paolo Ponzetto. 2012. BabelNet: The automatic construction, evaluation and application of a wide-coverage multilingual semantic network. Artificial Intelligence, 193:217-250.

Kishore Papineni, Salim Roukos, Todd Ward, and WeiJing Zhu. 2002. Bleu: a method for automatic evaluation of machine translation. In Proceedings of the 40th Annual Meeting of the Association for Computational Linguistics, pages 311-318, Philadelphia, Pennsylvania, USA. Association for Computational Linguistics.

Bolette S. Pedersen, Sanni Nimb, Jørg Asmussen, Nicolai Hartvig Sørensen, Lars Trap-Jensen, and Henrik Lorentzen. 2009. DanNet: the challenge of compiling a wordnet for Danish by reusing a monolingual dictionary. Language Resources and Evaluation, 43:269-299.

Emanuele Pianta, Luisa Bentivogli, and Christian Girardi. 2002. MultiWordNet: Developing an Aligned Multilingual Database. In Proceedings of the 1st International Conference on Global WordNet, pages 293-302.

Mohammad Taher Pilehvar and Jose CamachoCollados. 2019. WiC: the Word-in-Context dataset for evaluating context-sensitive meaning representations. In Proceedings of the 2019 Conference of the North American Chapter of the Association for Computational Linguistics: Human Language Technologies, Volume 1 (Long and Short Papers), pages 1267-1273, Minneapolis, Minnesota. 
Eli Pociello, Antton Gurrutxaga, Eneko Agirre, Izaskun Aldezabal, and German Rigau. 2008. WNTERM: Enriching the MCR with a terminological dictionary. In LREC 2008.

Marten Postma, Emiel van Miltenburg, Roxane Segers, Anneleen Schoen, and Piek Vossen. 2016. Open Dutch WordNet. In Proceedings of the Eight Global Wordnet Conference.

Quentin Pradet, Gaël de Chalendar, and Jeanne Desormeaux Baguenier. 2014. Wonef, an improved, expanded and evaluated automatic french translation of wordnet. In Proceedings of the Seventh Global Wordnet Conference (GWC2014), pages 32-39.

Peng Qi, Yuhao Zhang, Yuhui Zhang, Jason Bolton, and Christopher D. Manning. 2020. Stanza: A Python Natural Language Processing Toolkit for Many Human Languages. In In Association for Computational Linguistics (ACL) System Demonstrations.

Ida Raffaelli, Marko Tadić, Božo Bekavac, and Željko Agić. 2008. Building croatian wordnet. In Fourth global wordnet conference ( gwc 2008).

Alessandro Raganato, Jose Camacho-Collados, and Roberto Navigli. 2017. Word Sense Disambiguation: A Unified Evaluation Framework and Empirical Comparison. In Proc. of EACL, pages 99-110.

Ervin Ruci. 2008. On the current state of Albanet and related applications. Technical report, University of Vlora. (http://fjalnet.com/technicalreportalbanet. pdf).

Bianca Scarlini, Tommaso Pasini, and Roberto Navigli. 2020. With More Contexts Comes Better Performance: Contextualized Sense Embeddings for AllRound Word Sense Disambiguation. In Proc. of EMNLP.

Karin Kipper Schuler, Anna Korhonen, and Susan Brown. 2009. VerbNet overview, extensions, mappings and applications. In Proceedings of Human Language Technologies: The 2009 Annual Conference of the North American Chapter of the Association for Computational Linguistics, Companion Volume: Tutorial Abstracts, NAACL-Tutorials '09, page 13-14, USA. Association for Computational Linguistics.

Federico Scozzafava, Marco Maru, Fabrizio Brignone, Giovanni Torrisi, and Roberto Navigli. 2020. Personalized pagerank with syntagmatic information for multilingual word sense disambiguation. In Proc. of ACL, pages 37-46.

Mehrnoush Shamsfard, Akbar Hesabi, Hakimeh Fadaei, Niloofar Mansoory, Payam Noor, Ali Reza Gholi Famian, Somayeh Bagherbeigi, Elham Fekri, and Maliheh Monshizadeh. 2010. Semi automatic development of FarsNet; the Persian WordNet In Proceedings of 5th global WordNet conference.
Kiril Simov and Petya Osenova. 2010. Constructing of an Ontology-based Lexicon for Bulgarian. In Proceedings of the Seventh International Conference on Language Resources and Evaluation (LREC'10), Valletta, Malta. European Language Resources Association (ELRA).

Jörg Tiedemann. 2012. Parallel data, tools and interfaces in OPUS. In Proceedings of the Eighth International Conference on Language Resources and Evaluation (LREC'12), pages 2214-2218, Istanbul, Turkey. European Language Resources Association (ELRA).

Jörg Tiedemann and Santhosh Thottingal. 2020. OPUS-MT - Building open translation services for the World. In Proceedings of the 22nd Annual Conference of the European Association for Machine Translation (EAMT), Lisbon, Portugal.

Ashish Vaswani, Noam Shazeer, Niki Parmar, Jakob Uszkoreit, Llion Jones, Aidan N Gomez, Łukasz Kaiser, and Illia Polosukhin. 2017. Attention is all you need. In Advances in neural information processing systems, pages 5998-6008.

Kadri Vider and Heili Orav. 2002. Estonian WordNet and Lexicography. In Proceedings of the Eleventh International Symposium on Lexicography, pages $549-555$.

Alex Wang, Yada Pruksachatkun, Nikita Nangia, Amanpreet Singh, Julian Michael, Felix Hill, Omer Levy, and Samuel R. Bowman. 2019. SuperGLUE: A stickier benchmark for general-purpose language understanding systems. In Proceedings of NeurIPS.

Ae-Sun Yoon, Soon-Hee Hwang, Eun-Ryoung Lee, and Hyuk-Chul Kwon. 2009. Construction of Korean WordNet. Journal of KIISE: Software and Applications, 36(1):92-108.

\section{Appendix}

\section{A Comparison between Italian WordNet and Wiktionary datasets}

In Table 8 we provide a small comparison between the Italian WordNet and Wiktionary datasets, which is the only language that overlaps. While this comparison is quite limited, it provides a few hints on the qualitative differences between Wiktionary and WordNet datasets.

\section{B Models' Parameters}

In Table 9 we report the parameters of the models used in our experiments. 


\begin{tabular}{|c|c|c|c|}
\hline & & WordNet & Wiktionary \\
\hline \multirow{4}{*}{ 茝 } & Instances & 260 & 592 \\
\hline & Unique Words & 105 & 394 \\
\hline & Avg. Ctx length & 14.53 & 23.39 \\
\hline & Human acc. & 82.0 & 78.0 \\
\hline \multirow{3}{*}{$\begin{array}{l}\overrightarrow{0} \\
\frac{1}{0} \\
N\end{array}$} & XLM-R base & 66.15 & 55.91 \\
\hline & XLM-R large & 80.00 & 64.86 \\
\hline & mBERT & 70.00 & 58.61 \\
\hline
\end{tabular}

Table 8: Statistics and comparison between the Italian WordNet and the Italian Wiktionary WiC datasets. Zero-shot results are computed by using the original English WiC (Pilehvar and Camacho-Collados, 2019) for training and development.

\begin{tabular}{lr} 
Model & Trainable Parameters \\
\hline mBERT & $110 \mathrm{M}$ \\
BERT-large & $335 \mathrm{M}$ \\
XLMR-base & $270 \mathrm{M}$ \\
XLMR-large & $550 \mathrm{M}$ \\
\hline
\end{tabular}

Table 9: Number of parameters for our comparison systems.

\section{Additional experimental results}

WordNet datasets. Table 10 includes details on the variability of the results, in particular the average results from three runs, including the standard deviation, for the zero-shot cross-lingual setting this is the setting producing a higher variability in the results.

Wiktionary Datasets. In Table 11 we show the development and test results in the monolingual settings of the multilingual language models trained, tuned and tested on the XL-WiC language-specific datasets from Wiktionary.

Translation setting + Dictionary alignment. We include a setting where, after translating the English training set to each target language, we also retrieve the corresponding translation of the English target word through a multilingual dictionary. We use BabelNet (Navigli and Ponzetto, 2012) as multilingual dictionary for all languages, discarding the sentences where the translated target word could not be found. Table 12 shows the results.

\section{Translation models}

Translation models are trained using the MarianNMT framework (Junczys-Dowmunt et al., 2018) on a filtered version of all OPUS parallel corpora collection using a language identifier (CLD2). As hyper-parameters, each model is based on the base version of the Transformer architecture (Vaswani et al., 2017). All models and training details are available at https://github.com/Helsinki-NLP/ Opus-MT. To give an idea of the translation quality, Table 13 reports the BLEU scores (Papineni et al., 2002) for each model. We report the performance, as described within the Opus-MT project, on the latest available test sets from the series of WMT news translation shared tasks, or on $5 \mathrm{~K}$ sentences taken from either the Tatoeba corpus (Tiedemann, 2012), or the Bible corpus (Christodouloupoulos and Steedman, 2015):

- Bulgarian (BG): Tatoeba, model checkpoint $\mathrm{EN} \leftrightarrow \mathrm{BG}$ opus-2019-12-18

- Danish (DA): Tatoeba, model checkpoint $\mathrm{EN} \leftrightarrow \mathrm{DA}$ opus-2019-12-18

- Estonian (ET): newstest2018, model checkpoint $\mathrm{EN} \leftrightarrow \mathrm{ET}$ opus-2019-12-18

- Croatian (HR): Tatoeba, model checkpoint $\mathrm{EN} \rightarrow \mathrm{HR}$ opus-2019-12-04, HR $\rightarrow \mathrm{EN}$ opus2019-12-05

- Japanese (JA): bible-uedin, model checkpoint $\mathrm{EN} \rightarrow \mathrm{JA}$ opus-2020-01-08, JA $\rightarrow$ EN opus2019-12-18

- Dutch (NL): Tatoeba, model checkpoint $\mathrm{EN} \rightarrow \mathrm{NL}$ opus-2019-12-04, NL $\rightarrow \mathrm{EN}$ opus2019-12-05 


\begin{tabular}{|c|c|c|c|c|}
\hline Language & mBERT & XLMR-base & XLMR-large & Size \\
\hline BG & $58.72 \pm 1.60$ & $58.42 \pm 3.74$ & $\mathbf{6 4 . 7 0} \pm 2.05$ & 1220 \\
\hline DA & $63.26 \pm 2.25$ & $62.29 \pm 2.34$ & $\mathbf{6 8 . 9 1} \pm 2.45$ & 3406 \\
\hline ET & $59.40 \pm 2.74$ & $62.74 \pm 2.43$ & $\mathbf{6 8 . 2 9} \pm 2.97$ & 390 \\
\hline FA & $67.71 \pm 3.36$ & $64.67 \pm 5.07$ & $\mathbf{7 3 . 5 8} \pm 1.49$ & 800 \\
\hline HR & $65.93 \pm 2.25$ & $63.07 \pm 2.06$ & $\mathbf{6 8 . 6 3} \pm 3.20$ & 408 \\
\hline JA & $62.58 \pm 0.37$ & $59.63 \pm 3.11$ & $\mathbf{6 3 . 2 3} \pm 1.72$ & 824 \\
\hline $\mathrm{KO}$ & $61.21 \pm 2.34$ & $64.04 \pm 3.93$ & $\mathbf{6 9 . 4 0} \pm 3.61$ & 1014 \\
\hline NL & $63.55 \pm 1.37$ & $64.41 \pm 1.40$ & $\mathbf{7 2 . 1 4} \pm 1.88$ & 1004 \\
\hline $\mathrm{ZH}$ & $68.85 \pm 0.50$ & $61.69 \pm 3.78$ & $\mathbf{7 0 . 6 8} \pm 2.94$ & 5538 \\
\hline
\end{tabular}

Table 10: Zero-shot results on mBERT, XLMR-base and XLMR-large on the WordNet-based datasets when using the English WiC training and development sets.

\begin{tabular}{|c|c|c|c|c|c|c|c|c|c|}
\hline \multirow[b]{2}{*}{ Language } & \multicolumn{2}{|c|}{ mBERT } & \multicolumn{2}{|c|}{ XLMR-base } & \multicolumn{2}{|c|}{ XLMR-large } & \multicolumn{3}{|c|}{ Size } \\
\hline & Dev & Test & Dev & Test & Dev & Test & Train & Dev & Test \\
\hline $\mathrm{DE}$ & 79.73 & 81.58 & 78.93 & 80.84 & 83.03 & 84.03 & 48042 & 8870 & 24268 \\
\hline FR & 71.62 & 73.67 & 71.43 & 73.06 & 75.00 & 76.16 & 39428 & 8588 & 22232 \\
\hline IT & 73.23 & 71.96 & 75.25 & 68.58 & 74.24 & 72.30 & 1144 & 198 & 592 \\
\hline
\end{tabular}

Table 11: Results on mBERT, XLMR-base and XLMR-large on the Wiktionary-based datasets when using the language-specific training and development data.

\begin{tabular}{lcccccccccc}
\hline Model & EN & BG & DA & ET & FA & HR & JA & KO & NL & ZH \\
\hline All instances translated to & Target Language & Dictionary & Alignment & (Train: $:$ T-EN & - & Dev: T-EN) \\
\hline mBERT & - & 60.66 & 60.16 & $\mathbf{6 1 . 7 9}$ & - & $\mathbf{6 8 . 8 7}$ & 52.79 & - & 57.57 & - \\
XLMR-base & - & 57.30 & 57.34 & 51.79 & - & 59.80 & 51.70 & - & 60.26 & - \\
XLMR-large & - & $\mathbf{6 3 . 3 6}$ & $\mathbf{6 6 . 2 7}$ & 61.54 & - & 66.42 & $\mathbf{5 3 . 8 8}$ & - & $\mathbf{6 9 . 4 2}$ & - \\
L-BERT & - & 56.31 & 58.07 & 56.67 & - & 59.31 & 53.40 & - & 58.47 & - \\
\hline
\end{tabular}

Table 12: Results on the WordNet test sets when using automatically-translated data with a multilingual dictionaryalignment technique.

\begin{tabular}{ccccccc}
\hline Opus-MT & BG & DA & ET & HR & JA & NL \\
\hline EN $\rightarrow$ XX & 50.0 & 60.4 & 23.3 & 48.3 & 42.1 & 57.1 \\
$\mathrm{XX} \rightarrow$ EN & 59.4 & 63.6 & 30.3 & 58.7 & 41.7 & 60.9 \\
\hline
\end{tabular}

Table 13: BLEU score of the translation models. 